\title{
Choque refratário e óbito após intoxicação por sulfato ferroso
}

\author{
Refractory shock and death by iron poisoning \\ Marith Graciano Berber ${ }^{1}$, Livia Meirelles de Araújo', Cláudio Flauzino Oliveira² ${ }^{2}$ Eduardo Juan Troster ${ }^{3}$, Flávio Adolfo C. Vaz ${ }^{4}$
}

\section{RESUMO}

Objetivo: Descrever um caso de intoxicação por ferro em uma criança após ingestão acidental de sulfato ferroso.

Descrição do caso: Lactente de 11 meses de idade admitida após ingestão acidental de sulfato ferroso (dose desconhecida); apresentava diarréia, desidratação grave e torpor. Inicialmente, foram adotadas medidas para estabilização hemodinâmica, suporte ventilatório e terapia vasopressora. A dosagem de ferro sérico era de $259 \mu \mathrm{mol} / \mathrm{L}$, sendo iniciado desferoxamina. Apesar do tratamento, manteve quadro de instabilidade hemodinâmica, sem melhora após associação de adrenalina. Evoluiu para óbito 50 horas após admissão no hospital por choque refratário.

Comentários: O sulfato ferroso é uma medicação amplamente utilizada em nosso meio e de fácil acesso às crianças nos domicílios. No caso descrito, apesar do diagnóstico precoce e dos cuidados prestados prontamente, não foi possível evitar grave evolução para choque refratário e óbito. Portanto, é importante prevenir a intoxicação e conhecer a evolução de uma intoxicação por ferro, uma vez que o tratamento nem sempre evita a má evolução.

Palavras-chave: criança; sulfato ferroso; sobrecarga de ferro; choque.

\section{ABSTRACT}

Objective: To present a case of a child with iron intoxication due to an accidental ingestion of ferrous sulfate.

Case description: An eleven-month-old child was admitted to the hospital after an accidental ingestion of ferrous sulfate, presenting diarrhea, severe dehydration and drowsiness. Initially, therapies for hemodynamic stabilization, ventilatory support and vasopressor drugs were adopted. Laboratory test revealed high serum iron level $(259 \mu \mathrm{mol} / \mathrm{L})$ and deferoxamine administration was started. Despite treatment, the child persisted with hemodynamic instability, without improvement after epinephrine infusion and died 50 hours after admission, due to refractory shock.

Comments: The ferrous sulfate is widely used and easily accessible for children in their homes. In this case report, despite prompt diagnosis and early institution of adequate treatment, it was not possible to avoid the development of refractory shock and death. Therefore, it is important to avoid intoxication as well as to improve medical knowledge about the pathophysiology of iron intoxication, acknowledging that treatment not always avoids a poor outcome.

Key-words: child; ferrous sulfate; iron overload; shock.

\footnotetext{
${ }^{1}$ Residente do Departamento de Pediatria do Instituto da Criança (ICr) do Hospital das Clínicas (HC) da Faculdade de Medicina da Universidade de São Paulo (FMUSP), São Paulo, SP, Brasil

2Pós-graduando do Departamento de Pediatria do ICr/HC-FMUSP, São Paulo, SP, Brasil

${ }^{3}$ Professor livre docente pelo Departamento de Pediatria e coordenador do Centro de Terapia Intensiva Pediátrica do ICr/HC-FMUSP, São Paulo, SP, Brasil ${ }^{4}$ Professor titular de Pediatria e diretor do Centro de Terapia Intensiva Pediátrica do ICr/HC-FMUSP, São Paulo, SP, Brasil
}

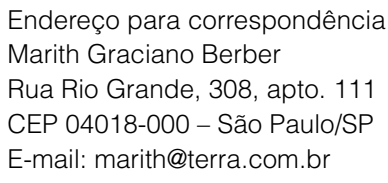

Recebido em: 27/4/2007 Aprovado em:31/7/2007 


\section{Introdução}

A ingestão excessiva de ferro é uma causa importante de intoxicação acidental na infância. O primeiro registro de intoxicação por ferro ocorreu na década de 1940, nos Estados Unidos. O uso de altas doses de ferro em comprimidos, utilizados em gestantes e no tratamento de doenças hematológicas, constitui a causa mais freqüente de óbito por intoxicação em crianças de um a quatro anos ${ }^{(1)}$. Em 1991, a incidência de intoxicação por ferro nos Estados Unidos foi de 18.450 casos, sendo a causa mais freqüente de intoxicação na infância. A maior parte dos casos ocorreu em crianças com menos de seis anos ${ }^{(2)}$.

Em 1997, o Food and Drug Administration iniciou campanhas preventivas e limitou em $30 \mathrm{mg}$ a concentração de ferro por comprimido. Desde então, houve queda considerável na incidência e na mortalidade da intoxicação pelo ferro: 29 óbitos foram registrados entre 1988 e 1997 e apenas um óbito ocorreu entre 1998 e 2002 . Além disso, os registros telefônicos caíram de 2,99/1.000 chamadas entre 1988 e 1997 para 1,99/1. 000 chamadas de 1998 e 2002 $2^{(3,4)}$.

No Brasil, como na maioria dos países, os medicamentos são os principais agentes tóxicos, respondendo por 28\% dos casos de intoxicação humana registrados anualmente, pelo Sistema Nacional de Informações Tóxico-Farmacológicas (Sinitox). Os benzodiazepínicos, os antigripais, antidepressivos e os antiinflamatórios são os medicamentos que mais intoxicam, sendo que as crianças menores de cinco anos representam cerca de $35 \%$ destas intoxicações ${ }^{(5)}$.

No caso descrito a seguir, apesar do diagnóstico precoce de intoxicação pelo sulfato ferroso e de cuidados adequadamente prestados, não foi possível evitar a grave evolução para choque refratário e óbito. O objetivo é discutir a evolução da intoxicação grave por ferro e destacar a necessidade do diagnóstico precoce e de ações preventivas.

\section{Descrição do caso}

Paciente de 11 meses, sexo feminino, sem antecedentes mórbidos, admitida na Unidade de Terapia Intensiva (UTI) após ingestão excessiva de sulfato ferroso.

Seis horas após a ingestão, iniciou quadro de vômitos e diarréia enegrecida, sendo trazida ao pronto-socorro com desidratação grave, rebaixamento do nível de consciência e má perfusão periférica. A mãe relatava ingestão acidental de sulfato ferroso pela criança, sem quantificar o número de comprimidos. Inicialmente, foram adotadas medidas para a estabilização hemodinâmica, sendo prescrita expansão de volume com cristalóide (três alíquotas de $20 \mathrm{~mL} / \mathrm{kg}$ em 20 minutos cada), intubação orotraqueal e suporte ventilatório, além de infusão contínua de dopamina (titulada até $15 \mathrm{mcg} / \mathrm{kg} / \mathrm{min}$ ). Após 12 horas da ingestão, foi dosado nível sérico de ferro de $259 \mu \mathrm{mol} / \mathrm{L}$ (valor de referência de 9 a $21,5 \mu \mathrm{mol} / \mathrm{L}$ ) e iniciada infusão contínua de desferoxamina $(15 \mathrm{mg} / \mathrm{kg} / \mathrm{hora})$. Apesar do tratamento, evoluiu com deterioração do quadro hemodinâmico e crise convulsiva tônico-clônica generalizada, controlada com benzodiazepínico intravascular. Mantinha instabilidade hemodinâmica, acidose metabólica e hiperglicemia, mesmo após nova reposição fluídica (duas alíquotas de $20 \mathrm{~mL} / \mathrm{kg}$ de cristalóide) e titulação da dopamina (até $20 \mathrm{mcg} / \mathrm{kg} / \mathrm{min}$ ), além de anemia, embora sem foco de sangramento aparente.

Foi transferida para a UTI em mau estado geral, descorada, hipotensa (PAM de $35 \mathrm{mmHg}$ ), Glasgow 3, pupilas isocóricas e fotorreagentes, pulsos não palpáveis, extremidades frias e edema em membros. Após a passagem de cateter venoso central, foi iniciada adrenalina, sendo titulada a dose de 0,2 a $4 \mathrm{mcg} / \mathrm{kg} / \mathrm{min}$, sem resposta. A pressão venosa central variou de 9 a $14 \mathrm{~cm}$ de água. Foram corrigidos distúrbios ácido-básicos e glicemia e foi realizada transfusão de hemoderivados. Evoluiu com anemia, coagulopatia, hipoproteinemia e choque refratário, falecendo 50 horas após a ingestão do sulfato ferroso (Tabela 1).

\section{Comentários}

A intoxicação pelo ferro geralmente ocorre em cinco etapas. No primeiro estágio, predominam os sintomas gastrintestinais, em geral, seis horas após a ingestão, como descrito no caso acima. O ferro é corrosivo à mucosa gástrica causando diarréia, náuseas, vômitos e sangramento gastrointestinal ${ }^{(6)}$. Apesar de a paciente não apresentar sangramento ativo evidente, provavelmente, a agressão à mucosa gastrintestinal causou perda de sangue, não evidenciada clinicamente pela rápida evolução do caso, justificando a queda tão significativa da hemoglobina. O segundo estágio, que ocorre entre seis e 24 horas, também é conhecido como latente, no qual aparecem acidose metabólica, hiperventilação e oligúria. Hiperglicemia e leucocitose também podem estar presentes, como observado no caso descrito. Neste momento, o uso de quelante do ferro pode evitar uma toxicidade maior. Se não é tomada uma conduta adequada, ocorre progressão para o terceiro estágio, caracterizado pelo choque refratário e falência hepática e renal, demonstrados 
pelo aumento significativo de aspartato-aminotranferase (AST), alanino-aminotransferase (ALT), uréia e creatinina. A falência múltipla de órgãos ocorre de 12 a 48 horas após a ingestão. Tanto a perda volêmica pelos vômitos e diarréia, quanto a toxicidade direta do ferro levam à necrose tubular aguda e falência hepática. No quarto estágio, há melhora clínica e, no quinto, o desenvolvimento de estenose do trato gastrintestinal, como complicação do efeito corrosivo do ferro sobre a mucosa.

Altas concentrações intracelulares de ferro resultam em disfunção mitocondrial e morte celular. A acidose metabólica causada pela intoxicação pelo ferro é resultado de diversos mecanismos. Depois de absorvido, o ferro é convertido da forma ferrosa para a férrica, liberando radicais de hidrogênio no plasma. A absorção do ferro pelos hepatócitos acarreta liberação de radicais livres e conversão para o metabolismo anaeróbio, com produção de ácido lático. Além disso, a presença de ferro livre no plasma causa aumento da permeabilidade capilar e perda do tônus venoso, o que culmina com o choque $e^{(7)}$.

As crianças que ingerem mais de $30 \mathrm{mg} / \mathrm{kg}$ de ferro elementar têm risco de intoxicação por ferro e devem ser investigadas. Os principais sintomas são enterorragia, choque, acidose metabólica com ânion gap elevado, disfunção hepática com aumento de AST, ALT, fosfatase alcalina e creatinoquinase (CK), leucocitose, necrose tubular aguda e mioglobinúria ${ }^{(6)}$. No caso, a mãe não soube quantificar a dose ingerida, mas o nível sérico do ferro confirmou a intoxicação.
Foi possível notar que a condução adequada do caso não foi capaz de prevenir o óbito e, mesmo com uso de desferoxamina, a criança evoluiu com choque refratário, indicando a necessidade de ações preventivas. Relatos de casos sugerem que a presença de acidose metabólica inexplicada e persistente pode ser o único sinal da intoxicação( ${ }^{(5)}$.

$\mathrm{Na}$ investigação, uma radiografia do abdome deve ser realizada. Se visualizados comprimidos de ferro no interior das alças, a lavagem intestinal está indicada. Se a medicação ainda estiver no estômago, deve-se realizar lavagem gástrica ou remoção por endoscopia.

Em pacientes assintomáticos, o nível sérico de ferro deve ser dosado após a quarta hora da ingestão. Quando o nível sérico do ferro for menor que $55 \mu \mathrm{mol} / \mathrm{L}$, não é necessária qualquer intervenção. Pacientes com nível sérico entre $55-90 \mu \mathrm{mol} / \mathrm{L}$ devem permanecer em observação por pelo menos 24 horas. Se, mesmo assim, permanecerem assintomáticos, nenhuma outra conduta específica deve ser tomada.

Pacientes com nível sérico de ferro superior a $90 \mu \mathrm{mol} / \mathrm{L}$ necessitam de tratamento intravenoso com desferoxamina. Os que apresentam sinais e sintomas sugestivos de falência de múltiplos órgãos necessitam de cuidados intensivos. Uma vez que a desferoxamina interfere no nível sérico do ferro, uma amostra de sangue deve ser coletada antes do início do tratamento. Entretanto, o mesmo não deve ser postergado na espera dos resultados ${ }^{(6)}$.

A desferoxamina quela o ferro livre e seus metabólitos são excretados na urina, conferindo-lhe uma coloração acastanha-

Tabela 1 - Progressão dos exames laboratoriais

\begin{tabular}{|c|c|c|}
\hline & 6 horas após ingestão & 48 horas após ingestão \\
\hline Hemoglobina/hematócrito & $16 \mathrm{~g} / \mathrm{dL} / 51 \%$ & $7 \mathrm{~g} / \mathrm{dL} / 21 \%$ \\
\hline Leucócitos & $45.000 / \mathrm{mm}^{3}$ & $1.400 / \mathrm{mm}^{3}$ \\
\hline Plaquetas & $210.000 / \mathrm{mm}^{3}$ & $83.000 / \mathrm{mm}^{3}$ \\
\hline AST & $3.502 \mathrm{U} / \mathrm{L}$ & $8.448 \mathrm{U} / \mathrm{L}$ \\
\hline ALT & $2.193 \mathrm{U} / \mathrm{L}$ & $5.428 \mathrm{U} / \mathrm{L}$ \\
\hline Lactato & $10 \mathrm{mmol} / \mathrm{L}$ & $167 \mathrm{mmol} / \mathrm{L}$ \\
\hline Ferro & $259 \mu \mathrm{mol} / \mathrm{L}$ & $144 \mu \mathrm{mol} / \mathrm{L}$ \\
\hline Uréia/creatinina & $86 / 1,0 \mathrm{mg} / \mathrm{dL}$ & $50 / 0,8 \mathrm{mg} / \mathrm{dL}$ \\
\hline Proteína total/albumina & $5,6 / 3,2 \mathrm{~g} / \mathrm{dL}$ & $3,4 / 2,5 \mathrm{~g} / \mathrm{dL}$ \\
\hline Gasometria & $\begin{array}{c}\mathrm{pH} 7,09 / \mathrm{pCO} 238,3 \\
\text { Bicarbonato } 11,9\end{array}$ & $\begin{array}{c}\mathrm{pH} 7,39 / \mathrm{pCO} 268 \\
\text { Bicarbonato } 42\end{array}$ \\
\hline Glicemia & $156 \mathrm{mg} / \mathrm{dL}$ & $22 \mathrm{mg} / \mathrm{dL}$ \\
\hline
\end{tabular}


da. Caso haja comprometimento significativo da função renal, esses metabólitos podem também ser removidos por meio de diálise. A desferoxamina deve ser mantida até que os sintomas desapareçam. Optar pela infusão contínua, iniciando-se com a dose de $10-15 \mathrm{mg} / \mathrm{kg} / \mathrm{h}$, sempre intravascular, uma vez que a droga é pouco absorvida após a administração oral ${ }^{(8)}$.

Diversos marcadores laboratoriais têm sido avaliados para determinar a toxicidade do ferro. A dosagem do nível sérico do ferro parece ser o melhor exame para determinar essa toxicidade. Entretanto, questiona-se se apenas a dosagem sérica pode determinar o risco de toxicidade. A dosagem da capacidade total de ligação do ferro (TIBC) é recomendada para estimar a presença de ferro livre e a necessidade do uso de quelante. O uso racional de TIBC é baseado na teoria de que o nível sérico de ferro se eleva

\section{Referências bibliográficas}

1. Tenenbein M. Unit-dose packaging of iron supplements and reduction of iron poisoning in young children. Arch Pediatr Adolesc Med 2005;159:557-60.

2. Watson WA, Litovitz TL, Rodgers GC Jr, Schwartz W, Reid N, Youniss J et al. 2004 Annual report of the American Association of Poison Control Centers Toxic Exposure Surveillance System. Am J Emerg Med 2005;23:589-666.

3. Woolf A, Litovitz T. Progress in the prevention of childhood iron poisoning. Arch Pediatr Adolesc Med 2005;159:593-5.

4. Morris CC. Pediatric iron poisonings in the United States. South Med J 2000;93:352-8.

5. Sinitox - Cict - Fiocruz [homepage on the Internet]. Envenenamento doméstico - Intoxicação infantil [cited 2007 May 27]. Available from: http://www. fiocruz.br/sinitox/medicamentos.htm. após a saturação de todas as proteínas carreadoras e que o ferro livre na circulação é um risco para a toxicidade e necessita de tratamento. Quando o TIBC for maior que o nível sérico, todo ferro está ligado à transferrina e não está disponível para causar toxicidade ${ }^{(9)}$.

O sulfato ferroso é uma medicação utilizada amplamente em nosso meio e de fácil acesso às crianças nos domicílios. Embora o risco da intoxicação seja grande, pode ser facilmente prevenida, por meio de orientação aos familiares e embalagens de medicamentos com trava de segurança. Além disso, uma vez que o uso de sulfato ferroso é amplamente difundido em nosso meio, deve-se estar atento aos sinais precoces de intoxicação pelo ferro para que seja tomada a conduta adequada, tentando evitar a evolução para choque refratário intratável.

6. Riordan M, Rylance G, Berry K. Poisoning in children: common medicines. Arch Dis Child 2002;87:400-2.

7. Black J, Zenel JA. Child abuse by intentional iron poisoning presenting as shock and persistent acidosis. Pediatrics 2003;111:197-9.

8. Molinoff GJ, Ruddon RW. Heavy metals and heavy-metal antagonists. In: Molinoff GJ, Ruddon RW, editors. Goodman \& Gilman's The pharmacological basis of therapeutics. $9^{\text {th }}$ ed. Texas: Mc Graw; 1995. p. 1649-69.

9. Siff $\mathrm{J}$ E, Meldon SW, Tomassoni AJ. Usefulness of the total iron binding capacity in the valuation and treatment of acute iron overdose. Ann Emerg Med 1999;33:73-6. 